\section{Molecular Syndromology}

Mol Syndromol 2017;8:272-277

DOI: $10.1159 / 000477750$
Accepted: May 12, 2017

by M. Schmid

Published online: July 1, 2017

\title{
Whole-Exome Sequencing Reveals FAT4 Mutations in a Clinically Unrecognizable Patient with Syndromic CAKUT: A Case Report
}

\author{
Amelie T. van der Ven ${ }^{a}$ Shirlee Shril ${ }^{a}$ Hadas Ityel $^{a} \quad$ Asaf Vivante $^{a} \quad$ Jing Chen $^{a}$ \\ Daw-Yang Hwang $^{a}$ Kristen M. Laricchiac ${ }^{c}$ Monkol Lek ${ }^{b, c}$ Velibor Tasic $^{d}$ \\ Friedhelm Hildebrandt ${ }^{a}$ \\ a Department of Medicine, Boston Children's Hospital, Harvard Medical School, and b Analytic and Translational \\ Genetics Unit, Massachusetts General Hospital, Boston, MA, and 'Program in Medical and Population Genetics, \\ Broad Institute of MIT and Harvard, Cambridge, MA, USA; ' Medical Faculty Skopje, University Children's Hospital, \\ Skopje, Macedonia
}

\section{Established Facts}

- Van Maldergem syndrome (VMS) is a rare genetic condition that is caused by recessive mutations in either DCHS1 (dachsous cadherin-related 1) or FAT4 (FAT atypical cadherin 4) genes.

- Very few publications have reported VMS cases with detailed phenotypic characterization and the particular molecular diagnosis to date.

- The published cases describe severe phenotypes including developmental delay, distinctive facial dysmorphism, auditory malformations with consecutive hearing loss, upper airway constriction as well as skeletal and hand malformations. Renal manifestations are frequently encountered.

\section{Novel Insights}

- We identified compound heterozygous mutations of FAT4 in a clinically unrecognizable, syndromic patient with symptoms including renal agenesis, mild facial dysmorphism, foot malformation, and scoliosis.

- Our findings for the first time suggest a variability of the classical VMS phenotype with a characteristic but significantly milder manifestation in comparison to previously reported cases.

\section{Key Words}

FAT4 · Syndromic CAKUT · Van Maldergem syndrome ·

Whole-exome sequencing

\section{Abstract}

We present the case of a patient of Macedonian origin with unilateral renal agenesis and ureterovesical junction obstruction in combination with further abnormalities includ- ing midface hypoplasia, scoliosis as well as camptodactyly of one toe. Whole-exome sequencing analysis revealed compound heterozygous variants in the FAT4 gene. Recessive variants in FAT4 are a known cause of van Maldergem syndrome (VMS) in which congenital anomalies of the kidney and urinary tract are a less characteristic but common feature. The initial presentation of our patient was not clinically recognizable. However, in view of the molecular findings, the most likely diagnosis is a mild manifestation of VMS. Only

\section{KARGER}

(C) 2017 S. Karger AG, Basel

E-Mail karger@karger.com

www.karger.com/msy
Friedhelm Hildebrandt, MD

Boston Children's Hospital, Division of Nephrology, EN561

300 Longwood Ave.

Boston, MA 02115 (USA)

E-Mail Friedhelm.Hildebrandt@ childrens.harvard.edu 
very few publications have reported patients with VMS and mutations in FAT4 to date. With this case, we hope to provide further insight into the phenotypic variability of this syndrome.

(c) 2017 S. Karger AG, Basel

Congenital anomalies of the kidney and urinary tract (CAKUT) are the main causes of chronic kidney disease in the first 3 decades of life [Vivante and Hildebrandt, 2016]. Despite the high prevalence and clinical significance only approximately $17 \%$ of isolated CAKUT cases can currently be explained by monogenic mutations in about 38 different genes [Sanna-Cherchi et al., 2012; Kohl et al., 2014; Vivante et al., 2017]. However, more than 200 monogenic syndromes are known to date that comprise CAKUT as part of their distinctive phenotype [Vivante and Hildebrandt, 2016].

\section{Methods}

We obtained blood samples, pedigree, and clinical information (http://www.renalgenes.org) from the respective probands. Whole-exome sequencing (WES) and data processing were performed by the Genomics Platform at the Broad Institute of Harvard and MIT (Broad Institute, Cambridge, MA, USA). Exome sequencing ( $>250 \mathrm{ng}$ of $\mathrm{DNA}$, at $>2 \mathrm{ng} / \mu \mathrm{L}$ ) was performed using Illumina exome capture (38-Mb target). Single nucleotide polymorphisms (SNPs) and insertions/deletions (indels) were jointly called across all samples using the Genome Analysis Toolkit (GATK) HaplotypeCaller. Default filters were applied to SNP and indel calls using the GATK Variant Quality Score Recalibration approach. Lastly, the variants were annotated using the Variant Effect Predictor. For additional information, please refer to the supplementary section 1 in the Exome Aggregation Consortium (ExAC) study [Lek et al., 2016]. The variant call set was uploaded on to seqr (https://seqr.broadinstitute.org) and analysis of the entire WES output was performed.

\section{Case Report and Results}

We here present a male with unilateral renal agenesis and contralateral ureterovesical junction obstruction of Macedonian origin (A4478-21). The patient also had facial dysmorphism with midface hypoplasia (Fig. 1a), cryptorchidism, mild to moderate developmental delay, hypotonia (neonatal), scoliosis (Fig. 1b) as well as camptodactyly of D3 of the left foot (Fig. 1c).

We performed a research-based SNP-genotyping array and WES in the DNA provided for patient A4478-21. Given the healthy parents, we assumed a recessive mode

FAT4 Mutations in Clinically

Unrecognizable Patient of inheritance. Using the seqr Software, we performed an untargeted analysis of our patient's WES output and identified 2 heterozygous variants in the FAT4 gene which segregated in trans (Fig. 1d). The maternally inherited mutation (c.9279A $>$ C, p.Gln3093His, rs769424345) yielded deleterious scores in the 3 in silico prediction programs used (PolyPhen2, SIFT, and Mutation Taster). The paternally inherited mutation (c.9313A $>$ G, p.Ser3105Gly, rs764097811) appeared less severe and was scored "deleterious" in only 1 of the 3 in silico prediction programs applied [NM_001291303.1]. The maternally inherited variant was conserved throughout all species listed in the Ensembl database (http://useast.ensembl.org/index. html). The paternally inherited variant was overall well conserved (41 out of 50 species in Ensembl). The amino acid substitution, p.Ser3105Gly, however, resembled the wild-type sequence of 2 species listed in the Ensembl database [NM_001291303.1].

Both identified variants have an extremely rare allele frequency in the general population, i.e., the maternally inherited variant has been reported 6 times heterozygously and never homozygously in approximately 60,000 individuals gathered in ExAC. The paternally inherited variant occurs 2 times heterozygously in the ExAC population and has never been reported homozygously. A screening of an in-house Macedonian cohort consisting of approximately 150 individuals was additionally conducted to investigate a potential regional augmentation of the observed FAT4 variants. The paternally inherited variant did not occur in this cohort, and the maternally inherited variant c.9279A $>$ C, p.Gln3093His was identified only in 1 individual within our cohort of approximately 150 Macedonians as a single heterozygous call, thereby providing further evidence against a significant local enrichment of our patient's variants in this population.

To exclude alternative, known explanations for our patient's presentation, we performed a targeted screening of the WES output of patient A4478-21 for all established monogenic causes for CAKUT in humans $(\sim 38)$ and mice $(>180)$ as well as all known approximately 200 monogenic causes for syndromic CAKUT without further findings. Additionally, all known CNVs for CAKUT as well as CNVs affecting regions known to harbor established CAKUT genes were excluded via SNP-genotyping array.

Recessive mutations in FAT4 have previously been identified in patients with van Maldergem syndrome (VMS) as well as Hennekam syndrome (HS) [Alders et al., 2014]. In their publication, Alders et al. [2014] provide

Mol Syndromol 2017;8:272-277 273 
a

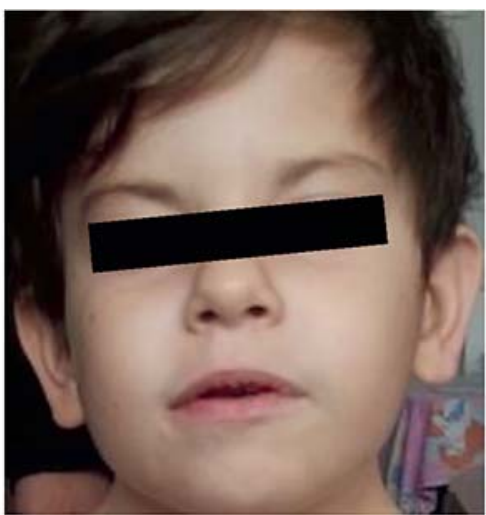

b

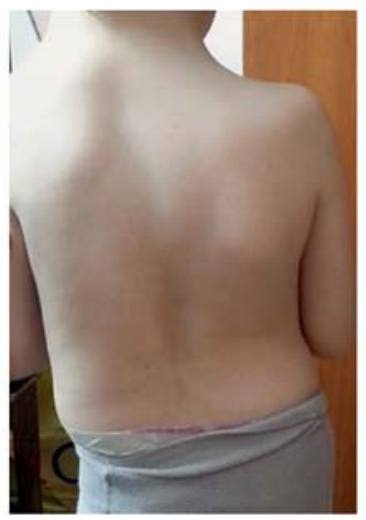

C

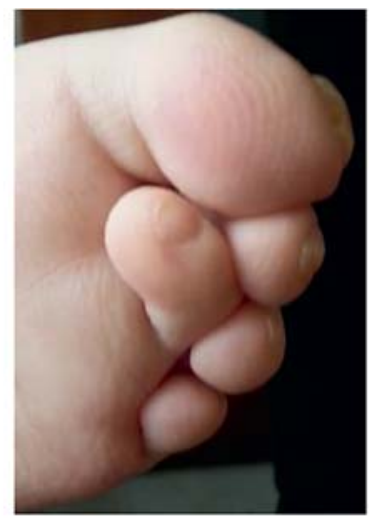

d

FAT4 Exon 9

FAT4 Exon 9 c.9279A $>C$ p.GIn3093His c.9313A $>$ G p.Ser3105Gly $\nabla$

Mother

Patient

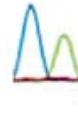

Father

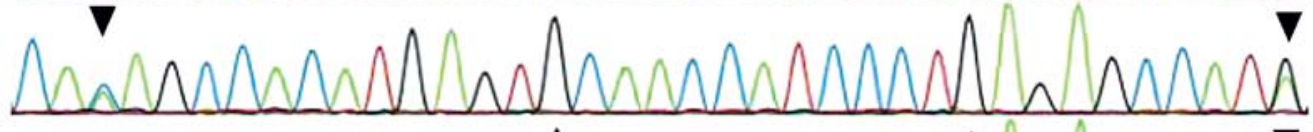

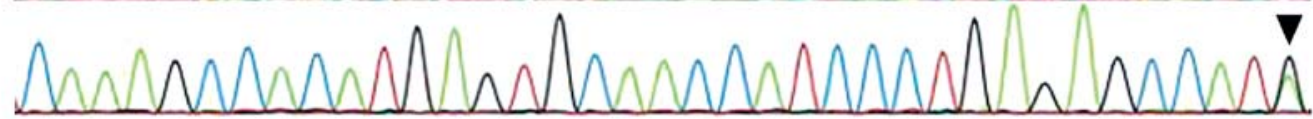

e Genotype/Phenotype relationship for FAT4 mutations in patients with Van Maldergem syndrome

\begin{tabular}{|c|c|c|c|c|c|c|c|c|c|c|c|c|c|c|c|c|c|c|c|}
\hline Identifier & Gene & $\begin{array}{l}\text { Zygosity of } \\
\text { affected } \\
\text { individuals }\end{array}$ & DNA & Protein & Exon & PP2 & SIFT & $\begin{array}{l}\text { Mut. } \\
\text { Tast. }\end{array}$ & PH & Deaf & CAKUT & $\begin{array}{c}\text { Hand } \\
\text { anomalies }\end{array}$ & $\begin{array}{c}\text { Tracheal } \\
\text { anomalies }\end{array}$ & $\begin{array}{l}\text { Skeletal } \\
\text { dysplasia }\end{array}$ & \begin{tabular}{|l|} 
Facial \\
dysmor \\
phism
\end{tabular} & $\mathrm{DD}$ & $\begin{array}{l}\text { Neonatal } \\
\text { hypotonia }\end{array}$ & Other & Publication \\
\hline \multirow[b]{2}{*}{ A4478-21 } & \multirow[b]{2}{*}{ FAT4 } & \multirow[b]{2}{*}{ comp. het } & c. $9313 A>G$ & $\begin{array}{c}\text { p.Ser3105 } \\
\text { Gly }\end{array}$ & 9 & 0.167 & $\begin{array}{l}\text { Del. } \\
(0)\end{array}$ & $\begin{array}{l}\text { Poly. } \\
\text { (1) }\end{array}$ & \multirow[b]{2}{*}{ ND } & \multirow[b]{2}{*}{ - } & \multirow[b]{2}{*}{+} & $(+)$ & \multirow[b]{2}{*}{ - } & \multirow[b]{2}{*}{$\begin{array}{c}\stackrel{(+)}{ } \\
\text { scoliosis }\end{array}$} & \multirow{2}{*}{$\begin{array}{c}(+) \\
\text { milder } \\
\text { than } \\
\text { classic } \\
\text { VMS }\end{array}$} & \multirow[b]{2}{*}{+} & \multirow[b]{2}{*}{+} & \multirow[b]{2}{*}{$\begin{array}{l}\text { cryptor } \\
\text { chidism }\end{array}$} & \multirow[b]{2}{*}{ this paper } \\
\hline & & & c. $9279 \mathrm{~A}>\mathrm{C}$ & $\begin{array}{c}\text { p. G } \ln 3093 \\
\text { His }\end{array}$ & 9 & 0.997 & $\begin{array}{l}\text { Del: } \\
(0)\end{array}$ & $\left|\begin{array}{c}\text { D.C. } \\
(0.999)\end{array}\right|$ & & & & $\begin{array}{c}\text { camptodact } \\
\text { yly of the } \\
\text { toe }\end{array}$ & & & & & & & \\
\hline \multirow{2}{*}{ F1 } & \multirow{2}{*}{ FAT4 } & \multirow{2}{*}{ comp. het. } & $\begin{array}{c}\text { c. } 12476 G> \\
T\end{array}$ & $\begin{array}{c}\text { p.Cys } 415 \\
\text { 9Phe }\end{array}$ & 14 & 0.182 & $\begin{array}{l}\text { Del. } \\
(0)\end{array}$ & D.c. (1) & \multirow{2}{*}{+} & \multirow{2}{*}{+} & \multirow{2}{*}{+} & \multirow{2}{*}{+} & \multirow{2}{*}{+} & \multirow{2}{*}{+} & \multirow{2}{*}{+} & \multirow{2}{*}{ ND } & \multirow{2}{*}{ ND } & \multirow{2}{*}{ ND } & \multirow{2}{*}{ [1] } \\
\hline & & & \begin{tabular}{|c|} 
c. $13193 G>$ \\
$A$
\end{tabular} & \begin{tabular}{|c|} 
p. Cys 439 \\
8 Tyr
\end{tabular} & 17 & 0.994 & $\begin{array}{l}\text { Del. } \\
(0)\end{array}$ & D.c. (1) & & & & & & & & & & & \\
\hline $\begin{array}{c}3-0095- \\
000\end{array}$ & FAT4 & hom. & c. $5275 A>G$ & $\begin{array}{c}\text { p.lle } 1759 \\
\text { Val }\end{array}$ & 2 & 0,963 & $\begin{array}{l}\text { Del. } \\
(0)\end{array}$ & D.c. (1) & ND & ND & ND & + & ND & + & + & + & + & $\begin{array}{c}\text { Autism, } \\
\text { hyposp } \\
\text { adias }\end{array}$ & [2] \\
\hline \multirow{2}{*}{ F2 } & \multirow{2}{*}{ FAT4 } & \multirow{2}{*}{ comp. het } & c. $7123 \mathrm{G}>\mathrm{A}$ & $\begin{array}{c}\text { p. Glu2375 } \\
\text { Lys }\end{array}$ & 7 & 0.998 & $\begin{array}{l}\text { Del. } \\
(0)\end{array}$ & D.c. (1) & \multirow[b]{2}{*}{ - } & \multirow[b]{2}{*}{+} & \multirow[b]{2}{*}{+} & \multirow[b]{2}{*}{+} & & & & & & & \\
\hline & & & c. $9481 \mathrm{G}>\mathrm{T}$ & p.Glu3161 & 9 & & & & & & & & + & + & ND & ND & ND & ND & [1] \\
\hline $\mathrm{F} 4$ & FAT4 & hom. & $\begin{array}{c}\text { c. } 11455 \mathrm{C}> \\
T\end{array}$ & p.Arg3819 & 9 & & & & + & NA & + & + & - & + & ND & ND & ND & ND & [1] \\
\hline F3 & FAT4 & hom. & \begin{tabular}{|c|} 
c. $14512 \_14$ \\
513 del
\end{tabular} & \begin{tabular}{|c|} 
p.Ser 4838 \\
Leufs*3 3
\end{tabular} & 17 & & & & - & + & + & + & + & NK & ND & ND & ND & ND & [1] \\
\hline
\end{tabular}

1

(For legend see next page.) 
a detailed comparison of the phenotypic features these 2 FAT4-related syndromes are characterized by. Generally, both syndromes demonstrate a broad phenotypic overlap, especially in regards to the facial dysmorphism (e.g., flat nasal bridge, small mouth, blepharophimosis) [Alders et al., 2014]. However, there are distinct features unique to either of the 2 syndromes that are not encountered in the other one. For example, features from the CAKUT spectrum are not present in their patient cohort of 9 patients with HS secondary to mutations in FAT4 when compared to 7 cases of FAT4-caused cases of VMS in which this is a regularly observed feature (5/7 patients) [Alders et al., 2014]. In comparison, defects in lymphatic structures resulting in congenital lymphedema of the limbs as well as internal organs, e.g., gut, lung, and kidneys occur in a majority of patients with HS (9/9 to 7/9 patients, respectively), whereas they are less frequently identified in patients with VMS (1/5) [Alders et al., 2014]. Online supplementary table 1 (for all suppl. material, see www.karger.com/doi/10.1159/000477750) lists the phenotypic features from both HS and VMS along with the manifestations encountered in patient A4478-21 with syndromic CAKUT.

On the basis of our findings, we performed a clinical ranking of previously published VMS cases (Fig. 1e). Figure 1e lists all of the so far reported variants in FAT4 along with information on the respective patient's phenotype and scores from prediction programs (scores based on transcript NM_001291303.1). Given the information

Fig. 1. Patient with syndromic CAKUT and mutations in FAT4. a Facial dysmorphism including a flat midface, broad nose with wide nasal bridge, tented upper lip. b Scoliosis. c Camptylodactyly with flexion deformity in D3 of the left foot. d Chromatograms obtained by direct Sanger sequencing of PCR products reveal compound heterozygous variants c.9279A $>C$ (resulting in p.Gln3093His) and c.9313A $>\mathrm{G}$ (resulting in p.Ser3105Gly) in exon 9 of the FAT4 gene in patient A4788-21 and in the heterozygous state in the parents, respectively. e Table listing recessive variants identified in FAT4 for patient A4478-21 and all previously reported patients with VMS and known molecular cause as well as sufficient phenotypic information $(n=6)$. The patients were ranked based on the deleteriousness of their least deleterious allele using 3 different prediction scores (Polyphen 2, SIFT, and Mutation Taster). Truncating mutations (either stop or frameshift with premature stop) did not yield prediction scores but were considered most deleterious. Prediction scores were calculated based on the information provided in the respective publications or based on transcript NM_001291303.1. DD, developmental delay; NA, not assessable; ND, no data; NK, not known; $\mathrm{PH}$, periventricular heterotopia. [1], Cappello et al., 2013. [2], Tammimies et al., 2015. provided, we were not confident to draw a conclusion regarding a potential genotype-phenotype correlation for FAT4-caused VMS cases. Looking at our patient, we however conclude that there is a variability of the "classic VMS phenotype" to what has been reported to date. This could potentially depend on the characteristics of the identified mutations.

Despite the milder presentation, the phenotypic overlap of our patient with VMS is undeniable in view of the genetic findings. We therefore tested whether the syndromic recognition program Face2Gene (https://app. face2gene.com) would have detected it. The Face2Gene result output did not include VMS or HS within the list of 30 suggested syndromes for facial images $(n=9)$ of our patient. On the contrary, the majority $(8 / 10)$ of the other reported cases of VMS with available images yielded a suggestion of HS within the first 3 results (online suppl. table 2). Known genetic causes for the 30 syndromes suggested in Face2Gene were excluded as potential cause of disease in individual A4478-21, if applicable (online suppl. table 3). Furthermore, an online search in the London Dysmorphology Database (http://suite.face2gene.com/ lmd-library-london-medical-database-dysmorphology/) using different combinations of terms describing the physical findings in our patient "developmental delay," "hypotonia," "midface hypoplasia," "scoliosis," "camptodactyly," "kidney agenesis" did not yield results including VMS or HS. Hence, it is very questionable whether the diagnosis of VMS would have ever been made without the molecular findings, given the relatively untypical presentation of patient A4478-21. This case therefore exemplifies the value of performing WES, especially in clinically ambiguous cases where alternative sources reach their limitations.

\section{Discussion}

VMS was first described in 1992 [Van Maldergem et al., 1992]. In 2013, Cappello et al. demonstrated that recessive mutations in the ligand-receptor pair DCHS1 and FAT4 are causative of 2 very similar manifestations of VMS. To date, there are 5 publications that provide detailed phenotypic descriptions of a total of 17 patients with VMS [van Maldergem et al., 1992; Zampino et al., 1994; Mansour et al., 2012; Neuhann et al., 2012; Cappello et al., 2013]. Only Cappello et al. [2013], however, provides phenotypic information and the molecular diagnosis for their patients (including 5 patients with mutations in FAT4). Ever since, 2 additional groups have been 
able to identify recessive mutations in FAT4 in single patients with phenotypic presentations reportedly compatible with a manifestation of VMS [Tammimies et al., 2015; Nolan and Carlson, 2016]. Detailed information on the phenotype, however, was only available for one of them [Tammimies et al., 2015].

Upon comparison, patient A4478-21 revealed many phenotypic characteristics compatible with a (mild) manifestation of VMS. Neonatal hypotonia and mild to moderate developmental delay are features present in the majority of reported cases with VMS [Mansour et al., 2012; Neuhann et al., 2012; Cappello et al., 2013]. Although much more subtle, our patient furthermore exhibits facial deformities that have previously been reported in the condition, including a flat midface, wide nasal bridge, tented upper lip, (Fig. 1a) mild ptosis, and thickened gums [Mansour et al., 2012; Neuhann et al., 2012; Cappello et al., 2013]. The scoliosis in our patient (Fig. 1b) can be interpreted as a skeletal malformation in line with VMS. Scoliosis has been reported in 2 out of 6 patients by Mansour et al. [2012] and was observed in the corresponding mouse model [Mao et al., 2011]. Most characteristically, our patient exhibited camptodactyly with fixed flexion deformity of D3 of the left foot (Fig. 1c). Camptodactyly is a commonly observed feature in VMS. Although usually present in the upper extremity, it has also been mentioned in the feet [Mansour et al., 2012].

Even though not part of the initial description [van Maldergem et al., 1992], the CAKUT phenotype is a common manifestation of VMS that has previously been reported [Mansour et al., 2012; Neuhann et al., 2012; Cappello et al., 2013]. Neuhann et al. [2012] furthermore described genital malformations, including cryptorchidism in their patient with VMS.

Not all the classic features of VMS were present in our patient, including, but not limited to, severe malformations, e.g., of the external auditory meatus with resulting hearing loss and upper airway constriction oftentimes requiring tracheostomy. Yet, it is striking that the very limited number of VMS cases in the literature can provide an explanation for all the phenotypic characteristics seen in our patient [Mansour et al., 2012; Neuhann et al., 2012; Cappello et al., 2013]. We therefore believe that the combination of our findings in WES, together with the phenotypic presentation of our patient is compatible with a mild manifestation of VMS.

Although VMS has exclusively been reported in patients with recessive mutations in either FAT4 or DCHS1, both genes are theoretically very intolerant to loss-offunction variants (probability of being loss-of-function intolerant score of 1 in ExAC, https://exac.broadinstitute. org) thereby implying a dominant mode of inheritance. Whether heterozygous mutations in FAT4 can generally cause human disease needs to further be investigated. No anomalies in the parents (i.e., heterozygotes) of the previously reported patients with recessive mutations in FAT4 have been mentioned. In our case, no irregularities suggestive of CAKUT were identified in the renal ultrasonogram of either of our patient's parents.

The mechanism by which variants in FAT4 contribute to the phenotypes reported in the literature remains to be elucidated. With our case, we hope to provide further insight into the phenotypic variability of VMS caused by mutations in FAT4. We furthermore aim to encourage physicians to consider WES in patients with CAKUT. As mentioned previously [Vivante et al., 2017] and further exemplified by this case, findings in WES can help clarify the diagnosis and teach us more about the phenotypic variability in patients with isolated or syndromic forms of CAKUT.

\section{Acknowledgments}

We thank the patient and their physician for the contribution of blood samples and clinical data. We furthermore thank Heidi Rehm, Daniel MacArthur, and Monkol Lek for their support with conducting WES, SNP-genotyping array, and analysis. This work was supported by grants from the National Institutes of Health (DK088767 to F.H. and HG008900 to Daniel MacArthur and Heidi Rehm). A.v.d.V. is supported by a Postdoctoral Research Fellowship from the German Research Foundation (VE916/1-1). A.V. is supported by a Fulbright postdoctoral scholar award and by grants from the Manton Center Fellowship Program, Boston Children's Hospital, Boston, and the Mallinckrodt Research Fellowship Award.

\section{Statement of Ethics}

Approval for experiments on human DNA was obtained from the Boston Children's Hospital Review Board. Informed consent was given from the respective probands.

\section{Disclosure Statement}

No author has competing interests to disclose. van der Ven et al. 


\section{References}

Alders M, Al-Gazali L, Cordeiro I, Dallapiccola B, Garavelli L, et al: Hennekam syndrome can be caused by FAT4 mutations and be allelic to Van Maldergem syndrome. Hum Genet 133: 1161-1167 (2014).

Cappello S, Gray MJ, Badouel C, Lange S, Einsiedler $\mathrm{M}$, et al: Mutations in genes encoding the cadherin receptor-ligand pair DCHS1 and FAT4 disrupt cerebral cortical development. Nat Genet 45:1300-1308 (2013).

Kohl S, Hwang DY, Dworschak GC, Hilger AC, Saisawat $\mathrm{P}$, et al: Mild recessive mutations in six Fraser syndrome-related genes cause isolated congenital anomalies of the kidney and urinary tract. J Am Soc Nephrol 25:19171922 (2014).

Lek M, Karczewski KJ, Minikel EV, Samocha KE, Banks E, et al: Analysis of protein-coding genetic variation in 60,706 humans. Nature 536: 285-291 (2016).

Mansour S, Swinkels M, Terhal PA, Wilson LC, Rich P, et al: Van Maldergem syndrome: further characterisation and evidence for neuronal migration abnormalities and autosomal recessive inheritance. Eur J Hum Genet 20. 1024-1031 (2012).
Mao Y, Mulvaney J, Zakaria S, Yu T, Morgan KM, et al: Characterization of a Dchs1 mutant mouse reveals requirements for Dchs1-Fat4 signaling during mammalian development. Development 138:947-957 (2011).

Neuhann TM, Müller D, Hackmann K, Holzinger S, Schrock E, Di Donato N: A further patient with van Maldergem syndrome. Eur J Med Genet 55:423-428 (2012).

Nolan D, Carlson M: Whole exome sequencing in pediatric neurology patients: clinical implications and estimated cost analysis. J Child Neurol 31:887-894 (2016).

Sanna-Cherchi S, Kiryluk K, Burgess KE, Bodria $\mathrm{M}$, Sampson MG, et al: Copy-number disorders are a common cause of congenital kidney malformations. Am J Hum Genet 91:987-997 (2012).
Tammimies K, Marshall CR, Walker S, Kaur G, Thiruvahindrapuram B, et al: Molecular diagnostic yield of chromosomal microarray analysis and whole-exome sequencing in children with autism spectrum disorder. JAMA 314: 895-903 (2015).

van Maldergem L, Wetzburger C, Verloes A, Fourneau C, Gillerot Y: Mental retardation with blepharo-naso-facial abnormalities and hand malformations: a new syndrome? Clin Genet 41:22-24 (1992).

Vivante A, Hildebrandt F: Exploring the genetic basis of early-onset chronic kidney disease. Nat Rev Nephrol 12:133-146 (2016).

Vivante A, Hwang DY, Kohl S, Chen J, Shril S, et al: Exome sequencing discerns syndromes in patients from consanguineous families with congenital anomalies of the kidneys and urinary tract. J Am Soc Nephrol 28:69-75 (2017).

Zampino G, Colosimo C, Balducci F, Mariotti P, Serra F, et al: Cerebro-facio-articular syndrome of Van Maldergem: confirmation of a new MR/MCA syndrome. Clin Genet 45: 140-144 (1994). 